\title{
Exponential decay of local conductance in single-wall carbon nanotubes
}

\author{
M. Stadermann, ${ }^{1}$ S. J. Papadakis, ${ }^{2}$ M. R. Falvo, ${ }^{3}$ Q. Fu, ${ }^{4}$ J. Liu, ${ }^{4}$ Y. Fridman, ${ }^{5}$ J. J. Boland,${ }^{6}$ \\ R. Superfine, ${ }^{2,3,5}$ and S. Washburn ${ }^{2,3,5,7}$ \\ ${ }^{1}$ Department of Chemistry, University of North Carolina at Chapel Hill, Chapel Hill, North Carolina 27599, USA \\ ${ }^{2}$ Department of Physics and Astronomy, University of North Carolina at Chapel Hill, Chapel Hill, North Carolina 27599, USA \\ ${ }^{3}$ Curriculum in Applied and Materials Science, University of North Carolina at Chapel Hill, Chapel Hill, North Carolina 27599, USA \\ ${ }^{4}$ Gross Chemistry Laboratory, Duke University, Box 90354, Durham, North Carolina 27708, USA \\ ${ }^{5}$ Department of Computer Science, University of North Carolina at Chapel Hill, Chapel Hill, North Carolina 27599, USA \\ ${ }^{6}$ Department of Chemistry, Trinity College, Dublin 2, Ireland \\ ${ }^{7}$ Department of Biomedical Engineering, University of North Carolina at Chapel Hill, Chapel Hill, North Carolina 27599, USA
}

(Received 13 September 2005; published 5 December 2005)

\begin{abstract}
We have measured the decay of local conductance in single-wall carbon nanotubes directly using conductance imaging atomic force microscopy. The decay lengths were in the range from $190 \mathrm{~nm}$ to well over $3 \mu \mathrm{m}$. There are strong indications that these decay lengths are the result of depletion lengths around metallic/ semiconducting carbon nanotube junctions, and that they are related to defects in the tubes.
\end{abstract}

DOI: 10.1103/PhysRevB.72.245406

PACS number(s): 73.63.Fg, 68.37.Ps, 73.63.Rt

\section{INTRODUCTION}

One-dimensional localization is an old area of theoretical study ${ }^{1,2}$ in which some incisive experiments have been performed. ${ }^{3}$ One-dimensional ballistic conductors also have been studied in theoretical ${ }^{4}$ and experimental ${ }^{5-8}$ investigations. In both limits (strong and weak disorder), screening and the decay of carrier wave functions have been emphasized. Since the first investigation of their electrical properties, single-wall carbon nanotubes (SW-CNTs) have been a promising candidate as one-dimensional building blocks for nanoscale circuitry. Thus, a multitude of measurements have been performed to characterize their conductance behavior. Almost all of these measurements have been performed with static contacts. Only few have been performed with mobile probes, ${ }^{9-11}$ and fewer still had high resolution., ${ }^{9,10}$ In the present study we will illustrate the utility of conductance imaging atomic force microscopy (CIAFM) in attacking these problems.

Specifically in this paper, we used CIAFM to analyze the local conductance of carbon nanotubes around nanotubenanotube junctions. We find that conductance decays exponentially in some cases, and that the decay lengths are dependent on the sample bias and back gate bias. Anticipating the ballistic character of the individual SW-CNT, ${ }^{12}$ we also study chemically etched SW-CNT. For a given CNT, each progressive etch process produces more defects and shortens the decay length as expected intuitively.

\section{DESIGN AND IMPLEMENTATION}

We will analyze the transport properties of the nanotubes by performing two-point measurements with a mobile probe on the nanotube. Initially, we use untreated chemical vapor deposition (CVD) tubes that should be in pristine condition. After each measurement step, we briefly etch the sample with piranha $\left(1: 3 \mathrm{H}_{2} \mathrm{O}_{2}: \mathrm{H}_{2} \mathrm{SO}_{4}\right)$ to increase the amount of defects in the tubes, and then measure again.
The samples used in our experiments consisted of nanotubes grown directly onto silicon dioxide substrates with CVD. ${ }^{13}$ On samples with back gates, aluminum was deposited on the back before electrodes where fabricated on the front, and then the sample was sintered for $30 \mathrm{~min}$ at $400{ }^{\circ} \mathrm{C}$ in vacuum. The oxide thickness was $1 \mu \mathrm{m}$. Two different methods of electrode deposition were used. The first type consisted of gold $(30 \mathrm{~nm})$ deposited onto the surface by thermal evaporation and patterned by shadow masking. On the second sample type, chrome $(10 \mathrm{~nm})$ and gold $(50 \mathrm{~nm})$ were patterned onto the front by photolithography. This type of sample was annealed at $600{ }^{\circ} \mathrm{C}$ for $10 \mathrm{~min}$ in vacuum after the metal deposition. The first sample type provides significantly lower contact resistances between tubes and electrodes, whereas the second sample type makes it easier to revisit a specific area of the sample after etching. The etching in this work was done with freshly prepared piranha, with a typical etch taking 60-120 s.

A conductance imaging microscope ${ }^{14}$ was used to take high-resolution conductance measurements on the nanotubes in contact with the electrode and spatially map the two-point resistance of the tubes. This AFM method uses a tuning fork force probe. A solid Pt/Ir tip is attached, which allows for simultaneous measurement of topography and conductance. The oscillation amplitude of the tuning fork was about $30 \mathrm{~nm}$ in every measurement, and scanning is performed with a frequency shift $\Delta f=1.5 \mathrm{~Hz}$. The force between tip and sample was estimated from frequency shift/distance curves to be on the order of $30 \mathrm{nN}$. Voltages of up to $\pm 1 \mathrm{~V}$ were applied to the sample, while the tip remained grounded. The tip shape can be deduced from a line analysis perpendicular to the nanotube. Using these traces, we find that the tip has an asperity at its end that has a radius of $60-80 \mathrm{~nm}$.

\section{RESULTS AND DISCUSSION}

The analysis of current change is always performed around junctions between two nanotubes, since this allows us 

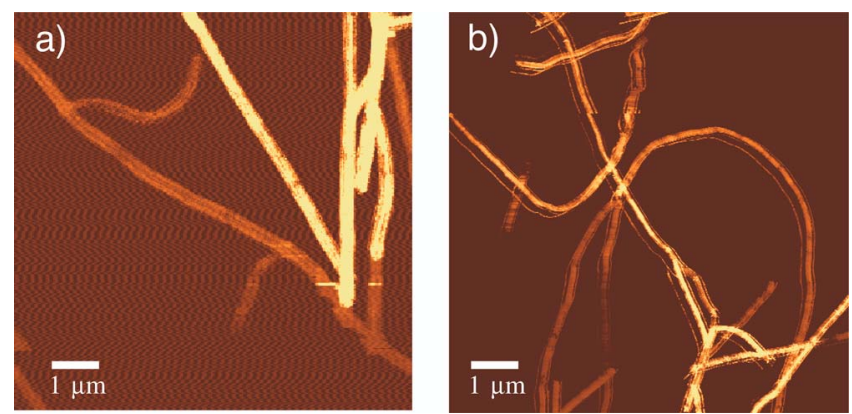

FIG. 1. (Color online) (a) Conductance image of untreated carbon nanotubes. (b) Conductance image of a nanotube network that was etched for $210 \mathrm{~s}$ in piranha.

to investigate changes that occur directly after the contact, whereas the tip size will prevent us from doing so on metaltube contacts where the metal layer is $30 \mathrm{~nm}$ thick. Thus, resistances measured are typically upward of $100 \mathrm{k} \Omega$, and changes of magnitudes below the noise level of about $5 \%$ will not be measurable. Due to signal processing, the absolute values gained from the measurement are lower than the true values for the conductance and should be considered lower boundaries for conductance.

The unetched CVD-grown samples show no change in current along their lengths, only sharp changes at the junctions, as shown before in Ref. 15. After the sample is etched with piranha, the changes in conductance around some junctions are no longer sharp, but decay gradually, as shown in Fig. 1.

We extract the conductance change by tracing the backbone of the nanotube in the image and then plotting the largest measured conductance versus the length (Fig. 2).

It can be ruled out immediately that the change in conductance is a tip artifact. The actual length scale on which the change in conductance takes place is significantly larger than any estimate of the tip radius, even for short length scales. The tip radius can, however, distort the relationship between conductance image and topography image, making it difficult to locate the point in the topographical image precisely where the change in conductance begins. A large tip can broaden the conductance signal near the junction where the tube slopes, since the point of contact between tip and tube changes as the tip moves off the junction. This problem does not exist far from the junction where the CNT lies flat on the substrate.

To extract a decay length $l$ from these data, the following equation was fit:

$$
G=B+A e^{-x / l} .
$$

In a first set of experiments, we compared the decay lengths measured on samples after etching for $120 \mathrm{~s}$ and then etching again for an additional $90 \mathrm{~s}$. We found that after the first etch, the decay lengths initially appear, and their length is reduced after the second etch step (Table I).

In a second set of experiments, we determined the behavior of the decay lengths when the sample bias is changed and when a back gate voltage is applied. Back gating the samples introduces two difficulties. First, the noise in the topographi-
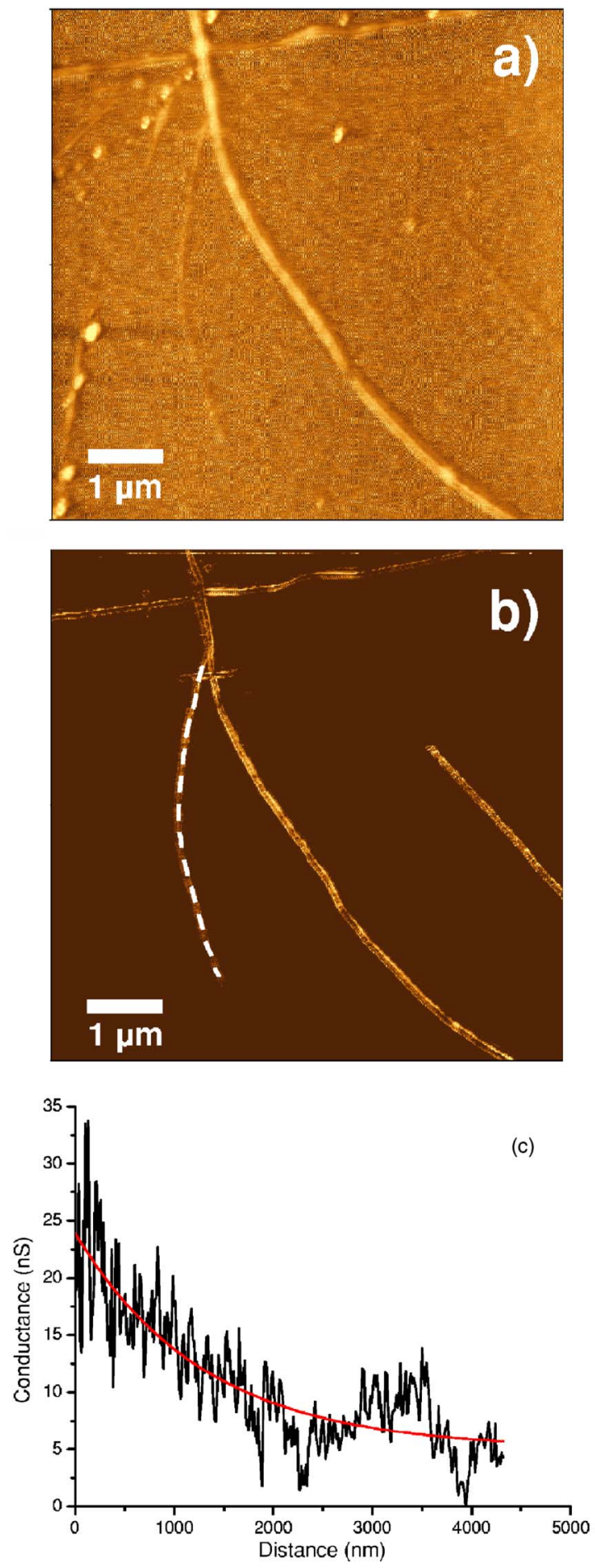

FIG. 2. (Color online) (a) Topography image of a nanotube junction. The noise in the image makes it difficult to determine the diameter of the tubes accurately. The bigger tube is estimated to be $2 \mathrm{~nm}$ in diameter, the smaller one below $1 \mathrm{~nm}$. (b) Conductance image of the junction taken simultaneously. The dotted white line indicates where the conductance trace shown in (c) was taken. (c) Trace along the small tube. The smooth line is an exponential decay fit to the data [see Eq. (1), with $A=19 \pm 0.7 \mathrm{nS}, B=5.0 \pm 0.6 \mathrm{nS}$, and $l=1290 \pm 140 \mathrm{~nm}]$. 
TABLE I. Summary of the decay lengths extracted from measurements on etched samples without back gate.

\begin{tabular}{ccc}
\hline \hline Sample & $\begin{array}{c}\text { Shortest decay } \\
\text { length } \\
(\mathrm{nm})\end{array}$ & $\begin{array}{c}\text { Longest decay } \\
\text { length } \\
(\mathrm{nm})\end{array}$ \\
\hline CVD, no etch & - & - \\
$\mathrm{CVD}, 120$ s etch & 1180 & 2250 \\
$\mathrm{CVD}, 210$ s etch & 180 & 890 \\
\hline \hline
\end{tabular}

cal image increases, and at the same time makes it more difficult to take clean conductance image. The amount of noise seems to be connected to the tip size, but we were unable to identify its source so far. Second, the decay length changes gradually towards an equilibrium value after every time the gate bias is changed (see Fig. 3), which is typically reached after about $80 \mathrm{~min}$. We believe that this is due to injection of charge into the substrate from the nanotube. ${ }^{16,17}$ This charge screens the gate voltage to some degree and changes the effective applied gate. This memory effect makes it difficult for us to tell what the exact gate voltage for each measurement is. We can, however, say with certainty that our measured decay lengths depend strongly on the applied gate voltages, where positive gate bias increases the decay length, and negative bias decreases the decay length as expected for charge density in a $p$-doped channel.

The decay length also changes if the source-drain bias (the bias between tip and sample) is changed. In all of the above measurements, the sample bias is $+1 \mathrm{~V}$, and the tip is held at $0 \mathrm{~V}$. When the sample bias is changed to $-1 \mathrm{~V}$, again with the tip at $0 \mathrm{~V}$, the decay length is reduced to approximately half of the length it had at positive bias. There appears to be no change in decay length over time after a change of the sample bias. This implies that the glassy effects mentioned above and in the literature are the result of charge motion in the oxide-not the CNT itself.

Conductance behavior as shown in Fig. 2 has been observed previously. ${ }^{11,18}$ Cumings $^{18}$ attributed this behavior to localization of electron wave functions. While the data in Table I suggest that the decay lengths in our experiments may be caused by the number of defects in our tubes, we almost never observe that the conductance goes to zero at long ranges. Instead, it usually reaches a finite value, indicating that localization is not the cause for the observed decay.

More consistent with our data would be that the observed decreases in conductance are caused by depletion lengths in semiconducting nanotubes around metallic/semiconducting contacts (Schottky barriers). For $p$-type semiconductors, which nanotubes in air are assumed to be, these depletion

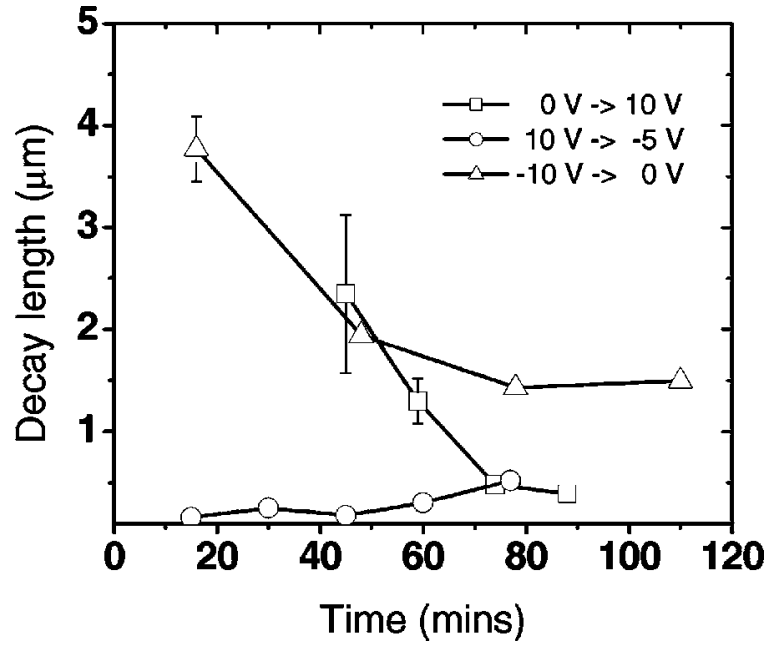

FIG. 3. Change of decay length after the gate bias is changed. In case of the squares and triangles, the gate bias was changed from a lower value to a higher value. The circles show a change from a high value to a lower value. The decay length seems to equilibrate after about $80 \mathrm{~min}$. The squares and the circles were measured sequentially on the same tube.

lengths should be larger when positive bias is applied to the back gate or the electrodes, and they should be smaller when negative biases are applied. An increase in depletion length would correspond to an increase in decay length. Both of these dependencies can be observed in our data. It is not certain what effect the piranha etch has on the electronic properties of tube in this case. The etching with piranha introduces carboxyl groups. ${ }^{19}$ We speculate that the high electronegativity of the oxygen in these groups reduces the electron density on the carbons, and thus these groups act as $p$-dopants. This assumption is consistent with the reduction of the decay length arising from increased carrier density if the decay is a result of screening.

\section{CONCLUSION}

We have measured the change of local conductance in carbon nanotubes around junctions between two nanotubes and found that it decays exponentially with decay lengths ranging between $190 \mathrm{~nm}$ and several microns. The decay length changes as we change the sample bias or the back gate bias, indicating that these decay lengths are connected to the depletion lengths around semiconducting/metallic junctions.

\section{ACKNOWLEDGMENTS}

This work was supported by the ONR-MURI program, as well as the National Science Foundation Ireland. 
${ }^{1}$ D. J. Thouless, Phys. Rev. Lett. 39, 1167 (1977).

${ }^{2}$ N. Mott and W. Twose, Adv. Phys. 10, 107 (1961).

${ }^{3}$ A. B. Fowler, A. Hartstein, and R. A. Webb, Phys. Rev. Lett. 48, 196 (1982).

${ }^{4}$ J. Voit, Rep. Prog. Phys. 58, 977 (1996).

${ }^{5}$ A. Yacoby, H. L. Stormer, N. S. Wingreen, L. N. Pfeiffer, K. W. Baldwin, and K. W. West, Phys. Rev. Lett. 77, 4612 (1996).

${ }^{6}$ F. P. Milliken, C. P. Umbach, and R. A. Webb, Solid State Commun. 97, R10653 (1996).

${ }^{7}$ M. Bockrath, D. Cobden, A. Rinzler, R. Smalley, L. Balents, and P. McEuen, Nature (London) 397, 598 (1999).

${ }^{8}$ H. W. C. Postma, M. de Jonge, Z. Yao, and C. Dekker, Phys. Rev. B 62, R10653 (2000).

${ }^{9}$ A. Bachtold, M. S. Fuhrer, S. Plyasunov, M. Forero, E. H. Anderson, A. Zettl, and P. L. McEuen, Phys. Rev. Lett. 84, 6082 (2000).

${ }^{10}$ M. Freitag, M. Radosavljević, W. Clauss, and A. T. Johnson, Phys. Rev. B 62, R2307 (2000).
${ }^{11}$ P. J. de Pablo, C. Gómez-Navarro, J. Colchero, P. A. Serena, J. G. Herrero, and A. M. Baró, Phys. Rev. Lett. 88, 036804 (2002).

${ }^{12}$ C. White and T. Todorov, Nature (London) 393, 240 (1998).

${ }^{13}$ Q. Fu, C. Lu, and J. Liu, Nano Lett. 2, 329 (2002).

${ }^{14}$ M. Stadermann, H. Grube, J. J. Boland, S. J. Papadakis, M. R. Falvo, R. Superfine, and S. Washburn, Rev. Sci. Instrum. 74, 3653 (2003).

${ }^{15}$ M. Stadermann, S. J. Papadakis, M. R. Falvo, J. Novak, E. Snow, Q. Fu, J. Liu, Y. Fridman, J. J. Boland, R. Superfine, and S. Washburn, Phys. Rev. B 69, 201402(R) (2004).

${ }^{16}$ M. S. Fuhrer, B. Kim, T. Dürkop, and T. Brintlinger, Nano Lett. 2, 755 (2002).

${ }^{17}$ M. Radosavljević, M. Freitag, K. V. Thadani, and A. T. Johnson, Nano Lett. 2, 761 (2002).

${ }^{18}$ J. Cumings and A. Zettl, Phys. Rev. Lett. 93, 086801 (2004).

${ }^{19}$ A. Kuznetsova, D. E. Mawhinney, V. Naumenko, J. T. Yates, J. Liu, and R. E. Smalley, Chem. Phys. Lett. 321, 292 (2000). 